\title{
ON TIDAL THEORIES AND THE ROTATION OF VISCOUS BODIES
}

\author{
G. Boué ${ }^{1}$, A.C.M. Correia ${ }^{1,2}$ and J. Laskar ${ }^{1}$
}

\begin{abstract}
Celestial bodies are complex systems with the ability to get deformed by tides. Modelling accurately this process is a difficult task even for the planets of our own solar system. This is why tidal theories applied to exoplanets are often reduced to a simple formula accounting for our lack of knowledge. In this work, we identify the three minimal assumptions leading to this level of simplification. We then compare different rheologies compatible with these hypotheses and discuss their qualitative behaviour. Finally, we apply the viscous model to close-in planets and describe their rotation evolution, emphasising the apparition of spin-orbit resonances as the viscous timescale increases.
\end{abstract}

\section{General comments on tidal theories}

We study the deformation of a celestial body by its rotation and by an external gravity field. Let us denote by $W(\vec{r}, t)$ the perturbing potential, i.e., the sum of the centrifugal and the external gravitational potentials, expressed in a body-fixed frame centred on the body's barycenter. The deformation is due to a differential acceleration within the body. The tide raising potential $\bar{W}_{T}(\vec{r}, t)$ is thus the remainder of the Taylor-McLaurin series of $W(\vec{r}, t)$ expanded at the first order

$$
\bar{W}_{T}(\vec{r}, t)=W(\vec{r}, t)-W(\overrightarrow{0}, t)-\vec{\nabla} W(\overrightarrow{0}, t) \cdot \vec{r} .
$$

The redistribution of material within the body induces a modification of its own gravitational potential $V(\vec{r}, t)$. If $V_{0}(\vec{r})$ denotes the potential without deformation, after perturbation, we get

$$
V(\vec{r}, t)=V_{0}(\vec{r})+V_{T}(\vec{r}, t)
$$

\footnotetext{
${ }^{1}$ IMCCE, Observatoire de Paris, UPMC Univ. Paris 6, PSL Research University, Paris, France

${ }^{2}$ CIDMA, Departamento de Física, Universidade de Aveiro, Campus de Santiago, 3810-193 Aveiro, Portugal

e-mail: gwenael.boue@obspm.fr; correia@ua.pt; jacques.laskar@obspm.fr
} 


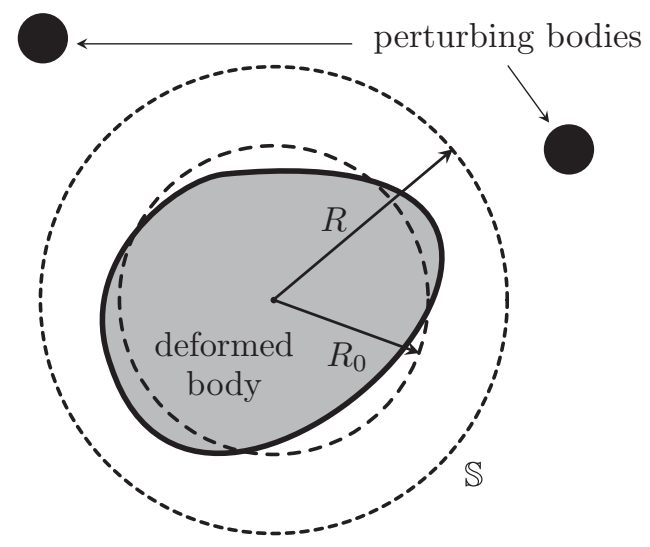

Fig. 1. Illustration of the system with the spheres of radius $R$ and $R_{0}$ involved in the calculation of the tidal equation.

where $V_{T}(\vec{r}, t)$ is the tidal potential. In a very general fashion, the tidal mechanism is described by a functional equation

$$
V_{T}=K\left(\bar{W}_{T}\right)
$$

where $K$ is an operator which depends only on the composition and on the physical properties of the body. A common assumption is to consider that $K$ is a linear operator, meaning that the total deformation is the sum of the tidal responses to each excitation. In that case, Equation (1.3) can be rewritten as

$$
V_{T}(\vec{r}, t)=\int K\left(\vec{r}, \vec{r}^{\prime}, t, t^{\prime}\right) \bar{W}_{T}\left(\vec{r}^{\prime}, t^{\prime}\right) \mathrm{d} \vec{r}^{\prime} \mathrm{d} t^{\prime}
$$

It sounds natural to restrict the domain of integration over $\vec{r}^{\prime}$ to the volume $\mathbb{V}$ of the body. If the body is incompressible, the integration can even be restricted to the surface $\partial \mathbb{V}$ without loss of information. Indeed, the centrifugal potential contains an isotropic term $W_{\text {iso }}\left(\vec{r}^{\prime}, t^{\prime}\right)$ which has no effect if the body is incompressible, and the rest of the tide raising potential $W_{T}\left(\vec{r}^{\prime}, t^{\prime}\right)=\bar{W}_{T}\left(\vec{r}^{\prime}, t^{\prime}\right)-W_{\text {iso }}\left(\vec{r}^{\prime}, t^{\prime}\right)$ satisfies Laplace equation $\Delta W_{T}=0$ inside $\mathbb{V}$ and remains finite at $\vec{r}^{\prime}=\overrightarrow{0}$. Thus the knowledge of $W_{T}$ at the surface $\partial \mathbb{V}$ of the body is sufficient to constrain $W_{T}\left(\vec{r}^{\prime}, t\right)$ everywhere inside $\mathbb{V}$. This situation corresponds to the Dirichlet problem. In practice, instead of the surface $\partial \mathbb{V}$, we choose a sphere $\mathbb{S}$ of radius $R$ centred on the barycenter and containing the full body (see Fig. 1). This may nevertheless not be possible if the external bodies raising the tides orbit very close to the surface. We discard this case in this study. Equivalently, $V_{T}$ satisfies Laplace equation everywhere beyond the surface $\mathbb{S}$ and remains finite as $\|\vec{r}\| \rightarrow \infty$, thus we only have to determine $V_{T}$ at the surface $\mathbb{S}$. Hence, we define a function $k$ such that

$$
V_{T}(R, \sigma, t)=\int k\left(R, \sigma, \sigma^{\prime}, t, t^{\prime}\right) W_{T}\left(R, \sigma^{\prime}, t^{\prime}\right) \mathrm{d} \sigma^{\prime} \mathrm{d} t^{\prime}
$$


where $\sigma=(\theta, \phi)$ are the coordinates (colatitude and longitude) of $\vec{r}^{\prime}$ on the sphere $\mathbb{S}$ and $\mathrm{d} \sigma=\sin \theta \mathrm{d} \theta \mathrm{d} \phi$. To proceed, we decompose $k\left(R, \sigma, \sigma^{\prime}, t, t^{\prime}\right)$ in spherical harmonics, i.e., we set

$$
\begin{aligned}
k\left(R, \sigma, \sigma^{\prime}, t, t^{\prime}\right) & =\sum_{l^{\prime}=0}^{\infty} \sum_{m^{\prime}=-l^{\prime}}^{l^{\prime}} k_{m^{\prime}}^{l^{\prime}}\left(R, \sigma, t, t^{\prime}\right) Y_{l^{\prime} m^{\prime}}^{*}\left(\sigma^{\prime}\right) \\
& =\sum_{l^{\prime}=0}^{\infty} \sum_{m^{\prime}=-l^{\prime}}^{l^{\prime}}\left(\sum_{l=0}^{\infty} \sum_{m=-l}^{l} k_{m m^{\prime}}^{l l^{\prime}}\left(R, t, t^{\prime}\right) Y_{l m}(\sigma)\right) Y_{l^{\prime} m^{\prime}}^{*}\left(\sigma^{\prime}\right)
\end{aligned}
$$

where $Y_{l^{\prime} m^{\prime}}^{*}\left(\sigma^{\prime}\right)$ denotes the complex conjugate of the spherical harmonic $Y_{l^{\prime} m^{\prime}}\left(\sigma^{\prime}\right)$. We also expand $V_{T}$ and $W_{T}$ in spherical harmonics as

$$
\begin{aligned}
& V_{T}(R, \sigma, t)=\sum_{l=0}^{\infty} \sum_{m=-l}^{l} V_{l m}(R, t) Y_{l m}(\sigma), \\
& W_{T}\left(R, \sigma^{\prime}, t^{\prime}\right)=\sum_{l^{\prime}=2}^{\infty} \sum_{m^{\prime}=-l^{\prime}}^{l^{\prime}} W_{l^{\prime} m^{\prime}}\left(R, t^{\prime}\right) Y_{l^{\prime} m^{\prime}}\left(\sigma^{\prime}\right) .
\end{aligned}
$$

The tide raising potential $W_{T}$ contains only harmonics of degree $l^{\prime} \geq 2$ because of its definition (Eq. (1.1)). Substituting expressions (1.6)-(1.8) into Equation (1.5), we obtain

$$
V_{l m}(R, t)=\sum_{l^{\prime}=2}^{\infty} \sum_{m^{\prime}=-l^{\prime}}^{l^{\prime}} \int k_{m m^{\prime}}^{l l^{\prime}}\left(R, t, t^{\prime}\right) W_{l^{\prime} m^{\prime}}\left(R, t^{\prime}\right) \mathrm{d} t^{\prime}
$$

This equation is not satisfying because the radius $R$ is arbitrary. To define a unique function $k_{m m^{\prime}}^{l l^{\prime}}\left(t, t^{\prime}\right)$, we make use of the radial dependence of $V_{l m}(R, t)$ and $W_{l m}\left(R, t^{\prime}\right)$. Because they both satisfy Laplace equation and remain finite when $R \rightarrow \infty$ and $R \rightarrow 0$ respectively, in the vicinity of $\mathbb{S}$ they behave as

$$
V_{l m}(R, t) \propto R^{-(l+1)}, \quad W_{l m}\left(R, t^{\prime}\right) \propto R^{l},
$$

which implies that $k_{m m^{\prime}}^{l l^{\prime}}\left(R, t, t^{\prime}\right) \propto R^{-(2 l+1)}$ in the vicinity of $\mathbb{S}$. Let $R_{0}$ be the mean radius of the body (see Fig. 1). We define $k_{m m^{\prime}}^{l l^{\prime}}\left(t, t^{\prime}\right)$ as the unique function such that

$$
k_{m m^{\prime}}^{l l^{\prime}}\left(R, t, t^{\prime}\right)=\left(\frac{R_{0}}{R}\right)^{2 l+1} k_{m m^{\prime}}^{l l^{\prime}}\left(t, t^{\prime}\right)
$$

for all $R$ between the surface of the body and any external masses. These functions $k_{m m^{\prime}}^{l l^{\prime}}\left(t, t^{\prime}\right)$ are called the Love distributions of the body. With this definition, let $V_{l m}(t)$ given by

$$
V_{l m}(t)=\sum_{l^{\prime}=2}^{\infty} \sum_{m^{\prime}=-l^{\prime}}^{l^{\prime}} \int k_{m m^{\prime}}^{l l^{\prime}}\left(t, t^{\prime}\right) W_{l^{\prime} m^{\prime}}\left(R_{0}, t^{\prime}\right) \mathrm{d} t^{\prime} .
$$


It should be noted that $V_{l m}(t)$ are not the spherical harmonic coefficients of the tidal potential at the radius $R_{0}$ since the sphere $\mathbb{S}$ of radius $R_{0}$ intersects the deformed body, and thus at this distance $V_{T}\left(R_{0}, \sigma, t\right)$ does not satisfy Laplace equation. Nevertheless, by construction, for all $r \geq R$, we do have

$$
V_{T}(r, \sigma, t)=\sum_{l=0}^{\infty}\left(\frac{R_{0}}{r}\right)^{l+1} \sum_{m=-l}^{l} V_{l m}(t) Y_{l m}(\sigma) .
$$

Thus far, to obtain the Equation (1.12), we only have assumed that the tidal response $V_{T}$ is linear with respect to the perturbing potential $W_{T}$. We now add the hypothesis of invariance by translation in time which means that $k_{m m^{\prime}}^{l l^{\prime}}\left(t, t^{\prime}\right)=$ $k_{m m^{\prime}}^{l l^{\prime}}\left(t+u, t^{\prime}+u\right)$ for all $u$. This simplification is valid as long as we focus on short term evolutions compared to geological timescales. Let $k_{m m^{\prime}}^{l l^{\prime}}(t) \equiv k_{m m^{\prime}}^{l l^{\prime}}(t, 0)$, choosing $u=-t^{\prime}$, we get

$$
V_{l m}(t)=\sum_{l^{\prime}=2}^{\infty} \sum_{m^{\prime}=-l^{\prime}}^{l^{\prime}} \int k_{m m^{\prime}}^{l l^{\prime}}\left(t-t^{\prime}\right) W_{l^{\prime} m^{\prime}}\left(R_{0}, t^{\prime}\right) \mathrm{d} t^{\prime},
$$

which is a convolution product. Naturally, $V_{l m}(t)$ should not depend on the values taken by the potential $W_{l^{\prime} m^{\prime}}$ in the future $t^{\prime}>t$, thus causality implies that $k_{m m^{\prime}}^{l l^{\prime}}(t)$ vanishes for all $t<0$. Equation (1.14) remains quite complicated as it mixes all the spherical harmonic degrees and orders. To reduce the number of parameters of the tidal model, we have to make an additional assumption. In particular, if we assume that the body is isotropic without perturbation, the tidal deformation must not depend on the orientation of the reference frame as long as it is fixed to the body. In that case, Schur's Lemma implies that there exists functions $k_{l}(t)$ such that

$$
k_{m m^{\prime}}^{l l^{\prime}}\left(t-t^{\prime}\right)=\delta_{l l^{\prime}} \delta_{m m^{\prime}} k_{l}\left(t-t^{\prime}\right)
$$

for all $\left(l, l^{\prime}, m, m^{\prime}\right)$ and where $\delta_{i j}$ is the Kronecker symbol equal to 1 if $i=j$, and 0 otherwise. Hence, with this hypothesis the tidal equation (Eq. (1.14)) reads

$$
V_{l m}(t)=\int k_{l}\left(t-t^{\prime}\right) W_{l m}\left(R_{0}, t^{\prime}\right) \mathrm{d} t^{\prime} .
$$

We stress that the last approximation is not valid for the Earth where the equator defines a privileged plane. In this frame, it is necessary to consider different functions $k_{l m}\left(t-t^{\prime}\right)$ to reproduce the evolution of the Earth spin axis, and in an arbitrary frame fixed to the planet all $k_{l m m^{\prime}}\left(t-t^{\prime}\right)$ are non zero.

In summary, the tidal equation (1.16) is valid for an incompressible body whenever the following three hypotheses hold true: 1) the body responds linearly to the excitation, 2) its structure is isotropic and 3) the evolution is short in comparison to geological timescales. In literature, Equation (1.16) is often written in the Fourier domain

$$
\hat{V}_{l m}(\omega)=\hat{k}_{l}(\omega) \hat{W}_{l m}\left(R_{0}, \omega\right)
$$

where $\hat{f}$ denotes the Fourier transform of $f$. 


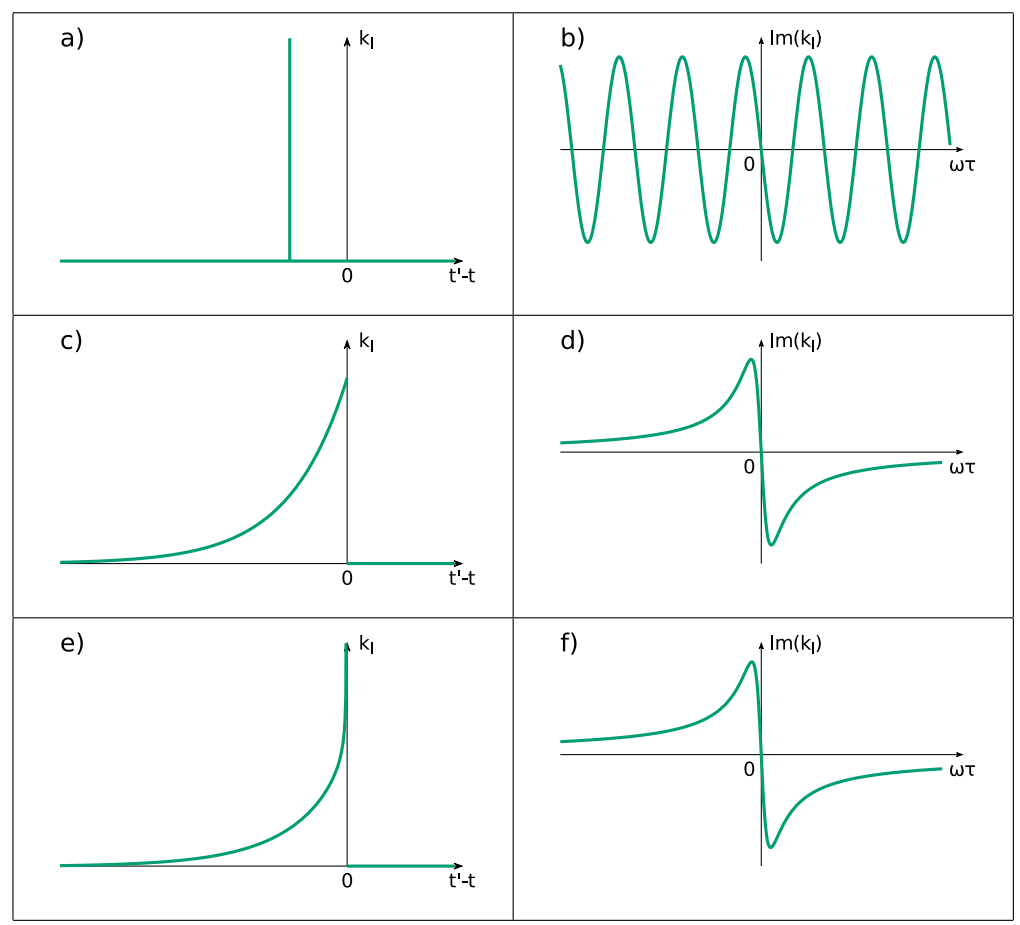

Fig. 2. Love distribution of the constant time lag model $(a-b)$, of the viscous model (c-d), and of Efroimsky's rheology (e-f) plotted in the temporal domain (left column) and in the Fourier domain (right column).

\section{Examples of rheology}

Above, we determined the general form of the tidal equation (Eq. (1.16)), but we did not discuss the expression of the Love functions $k_{l}\left(t-t^{\prime}\right)$. Causality implies that $k_{l}\left(t-t^{\prime}\right)=0$ for $t^{\prime}>t$. Moreover, the deformable body must only have a limited memory of the past and thus $k_{l}\left(t-t^{\prime}\right)$ must decrease to zero as $t-t^{\prime} \rightarrow \infty$. The evolution of $k_{l}\left(t-t^{\prime}\right)$ for $t-t^{\prime}$ between 0 and $+\infty$ depends on the composition and rheology of the body. Below we detail different models.

\subsection{Constant time lag}

The constant time lag model, introduced by Singer (1968), assumes that $k_{l}\left(t-t^{\prime}\right)$ is a Dirac distribution $\delta\left(t-t^{\prime}-\Delta t\right)$ such that

$$
V_{l m}^{\text {Singer }}(t)=W_{l m}\left(R_{0}, t-\Delta t\right)
$$

This distribution is plotted in Figure 2a. In the Fourier domain, as long as $\omega$ remains small in comparison to $2 \pi / \Delta t$, the imaginary part of $\hat{k}_{l}(\omega)$, which is 
proportional to the energy dissipated in tides, evolves linearly with $\omega$, and vanishes at $\omega=0$ (see Fig. 2b). This is expected as $\omega=0$ corresponds to a steady state where the deformation is permanent. There is thus no dissipation in that case. However, as shown in Figure 2b, at large frequencies the imaginary part of $\hat{k}_{l}(\omega)$ oscillates with a constant amplitude, meaning that very fast excitations are also able to dissipate energy. This is paradoxical because $\Delta t$ represents the amount of time required by the body to get deformed, but in this model if the perturbing potential evolves on a shorter timescale, after $\Delta t$ the body also gets deformed with the same timescale. This is a limitation of the model. A more realistic one should predict a decrease of dissipation as the perturbing frequency exceeds $2 \pi / \tau$ where $\tau$ is the response timescale.

\subsection{Viscous model}

According to the viscous - or Newton fluid - model (e.g., Ferraz-Mello 2013), the memory of the body decreases exponentially with time (see Fig. 2c). We have

$$
V_{l m}^{\text {viscous }}(t) \propto \int_{-\infty}^{t} \exp \left(-\frac{t-t^{\prime}}{\tau}\right) W_{l m}\left(R_{0}, t^{\prime}\right) \mathrm{d} t^{\prime} .
$$

This behaviour can be assimilated to a low-pass filter where the transfer function is of the form

$$
\hat{k}_{l}(\omega)=\frac{A}{1+\mathrm{i} \omega \tau} .
$$

Its imaginary part is represented in Figure 2d. With this model we recover the linear evolution of the imaginary part of $\hat{k}_{l}(\omega)$ at low frequency. Moreover, at high frequency the dissipation decreases to zero has expected. This model is thus qualitatively satisfying. Furthermore, this model can be put in the form of a differential equation

$$
V_{l m}^{\text {viscous }}(t)+\tau \dot{V}_{l m}^{\text {viscous }}(t)=A W_{l m}\left(R_{0}, t\right)
$$

which is very convenient to simulate systems of high eccentricities or with transient perturbations. The viscoelastic - or Maxwell - model is very similar. The memory of the body also decreases exponentially, but a Dirac distribution $\delta\left(t-t^{\prime}\right)$ is added to account for the instantaneous elastic response (e.g., Correia et al. 2014).

\subsection{Efroimsky rheology}

This model has been developed for terrestrial bodies like Earths and super-Earths by Efroimsky (2012). At low frequency, it assumes that these planets behave like a viscoelastic body with a smooth transition to higher frequency where the rheology is that of Andrade accounting for more dissipation. The model is naturally written in the Fourier domain

$$
\hat{V}_{l m}^{\text {Efroimsky }}(\omega)=A \frac{1+B(\mathrm{i} \omega \tau)^{-\alpha}+(\mathrm{i} \omega \tau)^{-1}}{C+B(\mathrm{i} \omega \tau)^{-\alpha}+(\mathrm{i} \omega \tau)^{-1}} \hat{W}_{l m}\left(R_{0}, \omega\right)
$$




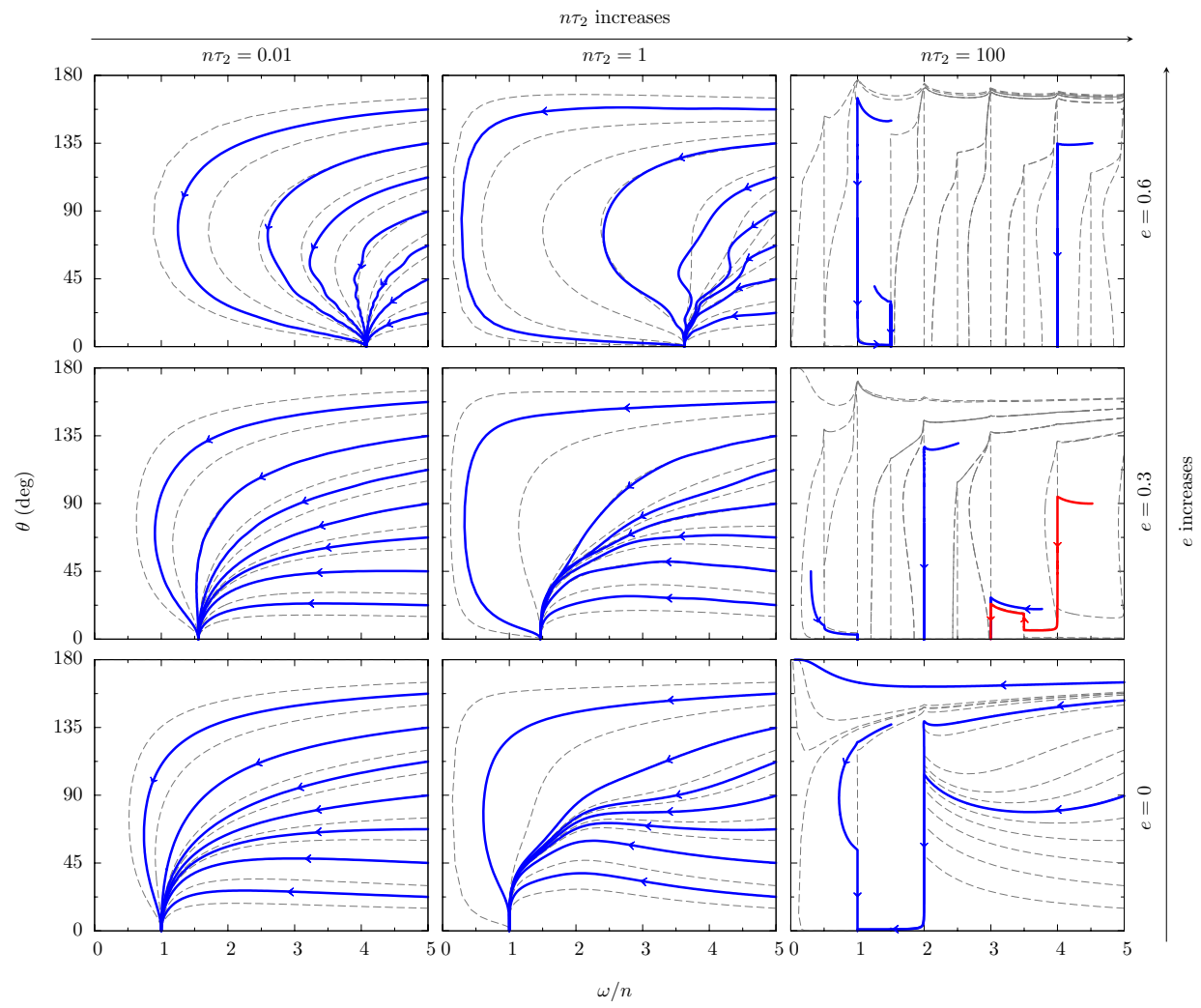

Fig. 3. Evolution of the spin axis of a short period planet for different eccentricities $e$ and viscous timescales $\tau_{2}$. The obliquity $\theta$ is displayed in the $y$-axis as a function of spin rate $\omega$ in unit of the orbital mean motion $n$. Dashed curved are the solutions of averaged equations, while solid curves are obtained by numerical integration of the instantaneous equations of motion.

where $B$ decreases exponentially when $\omega$ goes below the transition frequency. This model, plotted in Figure 2f, has the same qualitative characteristics as the viscous model except that the energy dissipation at high frequency decreases with a different power law. Note that the evolution provided in Figure 2e has been obtained numerically because there is no simple analytical expression in the time domain.

\section{Application}

To illustrate the importance of tides, we analyse the rotation behaviour of a short period planet deformed by its star. This study is taken from (Boué et al. 2016) where more details can be found. We assume that the planet satisfies the three hypotheses leading to the equation of tides (Eq. 1.16) and that its rheology is 
well described by the viscous model which has good qualitative properties and a convenient mathematical expression (see Sect. 2.2).

Results are displayed in Figure 3. When the viscous timescale is small compared to the orbital period, the evolution is well described by the constant time lag model: the obliquity decreases and the rotation speed gets capture in a pseudosynchronous state. But as the viscous timescale increases, several spin-orbit resonances arise. They appear in Figure 3 as vertical lines. In particular, we observe that the 0:1 and 2:1 spin-orbit resonances exist even in the case of a circular orbit as long as the obliquity is not nil.

\section{References}

Boué, G., Correia, A.C.M., \& Laskar, J., 2016, Celest. Mech. Dyn. Astron., 126, 31

Correia, A.C.M., Boué, G., Laskar, J., \& Rodríguez, A., 2014, Astron. Astrophys. J., $571, \mathrm{~A} 50$

Efroimsky, M., 2012, Astrophys. J., 746, 150

Ferraz-Mello, S., 2013, Celest. Mech. Dyn. Astron., 116, 109

Singer, S.F., 1968, Geoph. J. R. Astron. Soc., 15(1-2), 205 